\title{
The structure of thin accretion discs around magnetised stars
}

\author{
S. B. Tessema ${ }^{1,2}$ and U. Torkelsson ${ }^{2}$ \\ 1 Department of Physics, Addis Ababa University, PO Box 1176, Addis Ababa, Ethiopia \\ e-mail: newtonsolbel@yahoo.com \\ 2 Department of Physics, University of Gothenburg, SE 41296 Gothenburg, Sweden \\ e-mail: torkel@physics.gu.se
}

Received 13 July 2009 / Accepted 30 September 2009

\section{ABSTRACT}

\begin{abstract}
Aims. We determine the steady-state of an axisymmetric thin accretion disc with an internal dynamo around a magnetised star. Methods. Starting from the vertically integrated equations of magnetohydrodynamics we derive a single ordinary differential equation for a thin accretion disc around a massive magnetic dipole and integrate this equation numerically from the outside inwards. Results. Our numerical solution shows that the torque between the star and the accretion disc is dominated by the contribution from the dynamo in the disc. The location of the inner edge of the accretion disc varies between $R_{\mathrm{A}}$ and $10 R_{\mathrm{A}}$ depending mainly on the strength and direction of the magnetic field generated by the dynamo in the disc
\end{abstract}

Key words. accretion, accretion discs - magnetohydrodynamics (MHD) - magnetic fields - stars: neutron - X-rays: stars stars: pre-main sequence

\section{Introduction}

In this paper we present a new solution for an accretion disc around a magnetic star. This star could be a neutron star, a white dwarf, or a T Tauri-star, but we assume that it is a neutron star since it is easy to measure the torque between the neutron star and the accretion disc by timing the X-ray pulses from the neutron star. The new feature of our solution is to include the effect of an internal dynamo in the accretion disc. By doing this we hope to be able to explain the torque reversals that have been observed in some X-ray pulsars.

Shakura \& Sunyaev (1973) formulated the standard model of a geometrically thin, optically thick accretion disc. They were able to obtain an analytical solution of the heightintegrated hydrodynamic equations, after having introduced the $\alpha$-prescription for the turbulent stress, which transports the angular momentum outwards through the disc; however, they did not explain why the disc is turbulent in the first place, since a disc in Keplerian rotation is stable according to Rayleigh's criterion. Balbus \& Hawley (1991) instead showed that it is unstable if there is a weak magnetic field in the disc. Subsequent numerical simulations (e.g. Hawley et al. 1995; Balbus \& Hawley 1998) confirmed that this instability generates turbulence and that the resulting turbulent stresses transport angular momentum outwards.

The interaction between a magnetised star and a surrounding accretion disc is one of the most poorly understood aspects of accretion. At the same time, it is central for our understanding of the spin evolution of objects as diverse as T Tauri stars and X-ray pulsars. The magnetic field of the star penetrates the surrounding accretion disc and couples the two. According to the Ghosh \& Lamb (1979a,b) model, the part of the accretion disc that is located inside the corotation radius provides a spin up torque on the star, since it is rotating faster than the star, while the more slowly rotating outer part of the accretion disc brakes the star. The net torque is determined by the location of the inner edge of the disc, which moves inwards as the accretion rate increases, thereby increasing the spin up-torque on the star.

Campbell (1997) proposes physical descriptions of the magnetic diffusivity in terms of turbulence or buoyancy, and Campbell \& Heptinstall (1998a,b) solve the resulting equations numerically. For both forms of diffusivity, the magnetic coupling between the disc and the star leads to an enhanced dissipation in the inner part of the accretion disc compared to the standard Shakura \& Sunyaev (1973) model. This raises the temperature such that electron scattering dominates Kramer's opacity at larger radii than is otherwise the case, thus increasing the fraction of the disc that is subject to the (Lightman \& Eardley 1974) instability. Brandenburg \& Campbell (1998) considered a form of magnetic diffusivity that allows further analytical progress to be made, but the qualitative results remain the same.

All the models predict a positive correlation between the accretion rate and the torque on the neutron star and even predict a negative torque on the neutron star at very low accretion rates. Timing of X-ray pulsars during outbursts of Be/X-ray transients have provided at least some qualitative support for such a correlation (e.g. Parmar et al. 1989). The BATSE instrument on the Compton Gamma Ray Observatory made it possible to extend this database significantly (Bildsten et al. 1997). In particular there are a few X-ray pulsars with permanent discs that are oscillating between phases of constant spin-up and constant spindown without a significant difference in the X-ray luminosity between these states, which appears to contradict the standard model for a disc-accreting X-ray pulsar.

Nelson et al. (1997) propose that these torque-reversals can be the result of transitions between co-rotating and counterrotating accretion discs, though several other models have also been proposed. Torkelsson (1998) argue that the torque between an accreting star and its disc can be enhanced by the presence of a magnetic field generated by the turbulence in the accretion disc. The torque reversals are then the result of a reversal of the 
magnetic field generated by this dynamo. However, he did not construct a self-consistent model of the accretion disc. The aim of this paper is to construct such a model of an accretion disc with an internal dynamo around a magnetic star. We work in the spirit of Shakura \& Sunyaev (1973) and assume that the disc is geometrically thin. In Sect. 2 we start from the equations of magnetohydrodynamics (MHD) and derive a single ordinary differential equation for the radial structure of the accretion disc. We then present numerical solutions of this equation in Sect. 3 and discuss the properties of these solutions in Sect. 4. Finally we summarise our conclusions in Sect. 5.

\section{Mathematical formulation}

We study a steady, thin axisymmetric Keplerian disc around a star with a magnetic dipole field. The basic equations describing the structure of the thin accretion disc can be derived from the equations of magnetohydrodynamics.

\subsection{Conservation of mass}

In steady state the continuity equation takes the form

$\boldsymbol{\nabla} \cdot(\rho \boldsymbol{v})=0$

where $\rho$ is the density and $v=\left(v_{R}, v_{\phi}, v_{z}\right)$ is the fluid velocity with radial, azimuthal, and vertical components, respectively. For a thin axisymmetric disc and after neglecting a vertical outflow from the disc, we get

$\frac{1}{R} \frac{\partial}{\partial R}\left(R \Sigma v_{R}\right)=0$,

where $\Sigma$ is the surface density

$\Sigma=\int_{-H}^{H} \rho \mathrm{d} z \simeq 2 \rho H$,

and $H$ is the halfthickness of the disc. For a steady disc the integral of Eq. (2) gives

$\dot{M}=-2 \pi R \int_{-H}^{H} \rho v_{R} \mathrm{~d} z=-2 \pi R \Sigma v_{R}=$ constant,

which is the accretion rate.

\subsection{Conservation of momentum}

If assuming a steady state the Navier-Stoke's equation can be written as

$$
\begin{aligned}
\rho(\boldsymbol{v} \cdot \boldsymbol{\nabla}) \boldsymbol{v}= & -\boldsymbol{\nabla} P+\rho \boldsymbol{\nabla} \Phi+\boldsymbol{J} \times \boldsymbol{B} \\
& +\boldsymbol{\nabla} \cdot\left(\rho v\left(\boldsymbol{\nabla} \boldsymbol{v}-\frac{2}{3}(\boldsymbol{\nabla} \cdot \boldsymbol{v})\right)\right),
\end{aligned}
$$

where $P$ is pressure, $v$ kinematic viscosity, $\Phi$ the gravitational potential $\boldsymbol{J}=\frac{1}{\mu_{0}}(\boldsymbol{\nabla} \times \boldsymbol{B})=\left(J_{R}, J_{\phi}, J_{z}\right)$ the current density, and $\boldsymbol{B}=\left(B_{R}, B_{\phi}, B_{z}\right)$ the magnetic field. The viscosity is in general low, and we only retain it where it plays a crucial role.

The radial component of Navier- Stoke's equation is

$$
\begin{aligned}
\rho\left[v_{R} \frac{\partial v_{R}}{\partial R}-\frac{v_{\phi}^{2}}{R}\right]= & \frac{B_{\phi}}{\mu_{0}}\left(\frac{\partial B_{R}}{\partial z}-\frac{\partial B_{z}}{\partial R}\right)-\frac{B_{z}}{\mu_{0}}\left(\frac{1}{R} \frac{\partial}{\partial R}\left(R B_{\phi}\right)\right) \\
& -\frac{\partial P}{\partial R}-\frac{\rho G M R}{\left(R^{2}+z^{2}\right)^{3 / 2}} .
\end{aligned}
$$

For a thin accretion disc $v_{\phi} \gg c_{\mathrm{S}}$ as shown below and the dominant terms of the equation give us

$v_{\phi}^{2}-\frac{G M}{R}=0$

which shows that the disc rotates in a Keplerian fashion.

In similar manner, the vertical component of the momentum equation for a steady flow is

$$
\begin{aligned}
\rho\left[v_{R} \frac{\partial v_{z}}{\partial R}+v_{z} \frac{\partial v_{z}}{\partial z}\right]= & -\frac{B_{\phi}}{\mu_{0}} \frac{\partial B_{\phi}}{\partial z}-\frac{B_{R}}{\mu_{0}} \frac{\partial B_{R}}{\partial z}+\frac{B_{R}}{\mu_{0}} \frac{\partial B_{z}}{\partial R} \\
& -\frac{\partial P}{\partial z}-\frac{\rho G M}{R^{2}} .
\end{aligned}
$$

Neglecting vertical outflows and assuming the magnetic field to be weak the equation reduces to the equation of hydrostatic equilibrium

$\frac{1}{\rho} \frac{\partial P}{\partial z}=-\frac{G M}{R^{2}} \frac{z}{R}$

Using $H$ as the halfthickness of the disc, the pressure at the midplane of the disc is

$P=\frac{1}{2} H \Sigma \frac{G M}{R^{3}}$,

but the hydrostatic equilibrium can also be expressed as

$\frac{H}{R}=\frac{c_{\mathrm{s}}}{v_{\text {kepl }}}$

which shows that the Keplerian velocity is highly supersonic in a thin accretion disc, as assumed above.

The azimuthal component of Navier-Stoke's equation reduces to

$$
\begin{aligned}
\rho\left(\frac{v_{R}}{R} \frac{\partial}{\partial R}\left(R v_{\phi}\right)\right)= & \frac{B_{R}}{\mu_{0}} \frac{1}{R} \frac{\partial}{\partial R}\left(R B_{\phi}\right)+\frac{B_{z}}{\mu_{0}} \frac{\partial B_{\phi}}{\partial z} \\
& +\frac{1}{R^{2}} \frac{\partial}{\partial R}\left[R^{3} \rho v \frac{\partial}{\partial R}\left(\frac{v_{\phi}}{R}\right)\right] .
\end{aligned}
$$

We neglect $\frac{B_{R}}{R} \frac{\partial}{\partial R}\left(R B_{\phi}\right)$ because the radial length scale is much longer than the vertical length scale in a thin accretion disc. Integrating Eq. (12) vertically across the disc and multiplying both sides by $R$, we get

$\Sigma\left(v_{R} \frac{\partial l}{\partial R}\right)=\left[\frac{B_{z} B_{\phi}}{\mu_{0}}\right]_{-H}^{H} R+\frac{1}{R} \frac{\partial}{\partial R}\left[R^{3} v \Sigma \frac{\partial}{\partial R}\left(\frac{l}{R^{2}}\right)\right]$,

where the specific angular momentum $l=R v_{\phi} \propto R^{1 / 2}$. The magnetic term describes the exchange of angular momentum between the disc and the star via the magnetosphere. This term vanishes if $B_{\phi}$ is an even function of $z$, but the shear between the disc and the stellar magnetosphere generates an odd $B_{\phi}$ whose value in the upper half of the disc is

$B_{\phi, \text { shear }}=-\gamma B_{z} \frac{\Omega_{k}-\Omega_{\mathrm{s}}}{\Omega_{k}}$,

where $\Omega_{\mathrm{k}}=v_{\phi} / R, \Omega_{\mathrm{s}}$ is the angular velocity of the star, and $\gamma$ is a dimensionless parameter of a few (Ghosh \& Lamb 1979a,b).

In this paper we consider the effect of adding a largescale toroidal field that is generated by an internal dynamo in the accretion disc. Such a dynamo is a natural consequence of the magnetohydrodynamic turbulence in the accretion disc 
(e.g. Balbus \& Hawley 1998). To estimate the size of $B_{\phi, \text { dyn }}$, we assume for the moment that the viscous stress in the accretion disc is due to the internal magnetic stress

$f_{R \phi}=\frac{B_{R} B_{\phi, \mathrm{dyn}}}{\mu_{0}}=\alpha_{\mathrm{ss}} P$,

where we use the Shakura \& Sunyaev (1973) prescription for the viscosity in the last equality. Based on the results of numerical simulations of magnetohydrodynamic turbulence in accretion discs (e.g. Brandenburg et al. 1995) Torkelsson (1998) argues that

$\gamma_{\text {dyn }}=\frac{B_{\phi}}{B_{R}} \sim \frac{B_{\phi}}{B_{z}}$

where $\gamma_{\text {dyn }} \sim 10$. However, this $B_{\phi}$ is the sum of the large-scale field and a small-scale turbulent field, that is also contributing to the stress $f_{R \phi}$ through its correlation with a turbulent $B_{R}$-field. Since the large-scale field might be a small fraction of the total field we multiply $B_{\phi}$ with a factor $\epsilon$ to get an estimate for $B_{\phi, \text { dyn }}$ :

$B_{\phi, \mathrm{dyn}}=\epsilon\left(\alpha_{\mathrm{ss}} \mu_{0} \gamma_{\mathrm{dyn}} P\right)^{1 / 2}$,

where $-1 \leq \epsilon \leq 1$, and a negative value describes a magnetic field which is pointing in the negative $\phi$-direction at the upper disc surface.

We can now estimate the magnetic pressure in the accretion disc, to which the toroidal magnetic field is the main contributor. According to Eq. (17), the pressure is approximately

$\frac{B_{\phi, \text { dyn }}^{2}}{2 \mu_{0}}=\frac{1}{2} \epsilon^{2} \alpha_{\mathrm{ss}} \gamma_{\mathrm{dyn}} P$.

Since we use $|\epsilon| \leq 1, \alpha_{\mathrm{ss}}=0.01$ and $\gamma_{\mathrm{dyn}}=10$ in our models, we see that the magnetic pressure will not significantly affect the vertical structure of the accretion disc.

The vertical magnetic field can likewise be split up into two components; (i) the stellar dipolar magnetic field, whose value in the stellar equatorial plane is

$B_{z, \text { dipole }}=-\frac{\mu}{R^{3}}$,

where $\mu$ is the magnetic dipole moment; and (ii) a dynamo com-

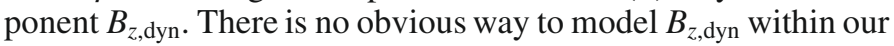
one-dimensional model, but numerical simulations like those by Brandenburg et al. (1995) suggest that $B_{z}$ and $B_{R}$ are comparable, so we expect that $B_{z \text {,dyn }} \sim B_{\phi \text {,dyn }} / \gamma_{\text {dyn }}$. In fact, $B_{z \text {,dyn will }}$ modify the structure of the poloidal magnetic field, but this can happen even if there is no internal dynamo in the disc because of the currents that are induced in the disc (e.g. Bardou \& Heyvaerts 1996).

We can now expand the product $B_{z} B_{\phi}$ as

$$
\begin{aligned}
B_{z} B_{\phi}= & \left(B_{z, \text { dipole }}+B_{z, \text { dyn }}\right)\left(B_{\phi, \text { shear }}+B_{\phi, \text { dyn }}\right) \\
= & B_{z, \text { dipole }} B_{\phi, \text { shear }}+B_{z, \text { dipole }} B_{\phi, \text { dyn }} \\
& +B_{z, \text { dyn }} B_{\phi, \text { shear }}+B_{z, \text { dyn }} B_{\phi, \text { dyn } .}
\end{aligned}
$$

As we see below the term $B_{z \text {,dipole }} B_{\phi \text {,dyn }}$ is significantly greater than $B_{z \text {,dipole }} B_{\phi \text {,shear }}$ almost everywhere in the disc. The term $B_{z \text {,dyn }} B_{\phi \text {,shear }}$ is smaller in size than $B_{z \text {,dipole }} B_{\phi \text {,dyn }}$ by a factor $\gamma / \gamma_{\text {dyn }}$, and since it is difficult to model it, we ignore it. Finally the term $B_{z \text {,dyn }} B_{\phi \text {,dyn }}$ does not contribute directly to the exchange of angular momentum between the accretion disc and the accretor, though it does affect it indirectly by contributing to the radial transport of angular momentum through the disc. Although this term can be important, we have decided to ignore it since there is no obvious way to model it in our onedimensional approach. One should notice here that this does not change the main qualitative conclusion of this paper that an internal dynamo in the accretion disc makes a significant contribution to the exchange of angular momentum between the disc and the star; on the contrary, we would see a stronger effect if we were to keep the $B_{z, \text { dyn }} B_{\phi, \text { dyn }}$-term. We now expand the $B_{z} B_{\phi} / \mu_{0}$ term in Eq. (13)

$$
\begin{aligned}
\Sigma\left(v_{R} \frac{\mathrm{d} l}{\mathrm{~d} R}\right)= & 2 \frac{B_{z}}{\mu_{0}} \epsilon\left(\alpha_{\mathrm{ss}} \mu_{0} \gamma_{\mathrm{dyn}} P\right)^{1 / 2} R-2 \gamma \frac{B_{z}^{2}}{\mu_{0}} \frac{\Omega_{k}-\Omega_{\mathrm{s}}}{\Omega_{k}} R \\
& +\frac{1}{R} \frac{\mathrm{d}}{\mathrm{d} R}\left[R^{3} v \Sigma \frac{\mathrm{d}}{\mathrm{d} R}\left(\frac{l}{R^{2}}\right)\right] .
\end{aligned}
$$

\subsection{Conservation of energy}

For a slow inflow of matter through an optically thick disc, the local viscous dissipation $\boldsymbol{v} \cdot \boldsymbol{f}_{v}$ is balanced by the radiative losses $\boldsymbol{\nabla} \cdot \boldsymbol{F}_{\text {rad }}$. This gives us

$\frac{9}{8} v \Sigma \frac{G M}{R^{3}}=\frac{4 \sigma T_{\mathrm{c}}^{4}}{3 \tau}$

where $T_{\mathrm{c}}$ is the temperature at the midplane of the disc, and $\sigma$ the Stefan-Boltzmann constant. The optical depth of the disc is given by

$\tau=\rho H \kappa_{\mathrm{R}}=\frac{1}{2} \Sigma \kappa_{\mathrm{R}}$,

and we assume that the opacity is given by Kramer's law

$\kappa_{\mathrm{R}}=\kappa_{0} \rho T_{\mathrm{c}}^{-7 / 2} \mathrm{~m}^{2} \mathrm{~kg}^{-1}$,

where $\kappa_{0}=5 \times 10^{20} \mathrm{~m}^{5} \mathrm{~kg}^{-2} \mathrm{~K}^{-7 / 2}$.

Equation (22) should also contain a term describing the magnetic dissipation

$2 H \frac{J^{2}}{\sigma}=\frac{2 B_{\phi}^{2}}{\sigma \mu_{0}^{2} H}=\frac{2 \epsilon^{2} \alpha_{\mathrm{ss}} \gamma_{\mathrm{dyn}} \eta P}{H}$,

where $\eta=1 / \sigma \mu_{0}$ is the magnetic diffusivity, and we have used Eq. (17) in the last equality. This should be compared to

$\frac{9}{8} v \Sigma \frac{G M}{R^{3}}=\frac{9}{4} \frac{v P}{H}$

so we see that the magnetic dissipation is negligible as long as $\eta \approx v$.

\subsection{Structure equations}

We reduce these equations to a single ordinary differential equation for the radial structure of the accretion disc. First we assume the equation of state of an ideal gas,

$P=\frac{\rho k_{\mathrm{B}} T_{\mathrm{c}}}{m_{\mathrm{p}} \bar{\mu}}$

where $k_{\mathrm{B}}$ is the Boltzmann constant, $\bar{\mu}$ the mean molecular weight, and $m_{\mathrm{p}}$ the mass of a proton, but the pressure can also be expressed using the equation of hydrostatic equilibrium

$\frac{1}{2} \frac{\Sigma G M H}{R^{3}}=\frac{\rho k_{\mathrm{B}} T_{\mathrm{c}}}{\bar{\mu} m_{\mathrm{p}}}$ 
which gives us a relation between $H$ and $T_{\mathrm{c}}$

$H=\left(\frac{k_{\mathrm{B}}}{m_{\mathrm{p}} \bar{\mu} G M}\right)^{1 / 2} T_{\mathrm{c}}^{1 / 2} R^{3 / 2}$.

The viscous stress tensor gives us the equation

$f_{\mathrm{r} \phi}=\frac{3}{4} \Sigma v\left(\frac{G M}{R^{3}}\right)^{1 / 2} H^{-1}=\alpha_{\mathrm{ss}} P(r)$,

which we solve for the density of the gas

$\rho=\frac{3}{4} \alpha_{\mathrm{ss}}^{-1}\left(\frac{m_{\mathrm{p}} \bar{\mu}}{k_{\mathrm{B}}}\right)^{3 / 2} \frac{v \Sigma}{T_{\mathrm{c}}^{3 / 2} R^{3}} G M$.

The optical depth of the disc is

$\tau=\kappa_{0} \frac{9}{16} \alpha_{\mathrm{ss}}^{-2}\left(\frac{m_{\mathrm{p}} \bar{\mu}}{k_{\mathrm{B}}}\right)^{5 / 2}(G M)^{3 / 2}(v \Sigma)^{2} T_{\mathrm{c}}^{-6} R^{-9 / 2}$.

Using Eqs. (22) and (32) we get

$T_{\mathrm{c}}=C \bar{\mu}^{1 / 4} \alpha_{\mathrm{ss}}^{-1 / 5} M^{1 / 4} y^{3 / 10} R^{-3 / 4}$,

where $y=v \Sigma$ and

$C=\left(\frac{243 \kappa_{0}}{512 \sigma}\right)^{1 / 10}\left(\frac{G m_{\mathrm{p}}}{k_{\mathrm{B}}}\right)^{1 / 4}$.

The pressure is then given by

$P=C_{1} \bar{\mu}^{3 / 8} \alpha_{\mathrm{ss}}^{-9 / 10} M^{7 / 8} y^{17 / 20} R^{-21 / 8}$,

where

$C_{1}=\frac{3}{4} G^{1 / 2}\left(\frac{243 \kappa_{0}}{512 \sigma}\right)^{-1 / 20}\left(\frac{G m_{\mathrm{p}}}{k_{\mathrm{B}}}\right)^{3 / 8}$.

The $\phi$-component of the magnetic field generated by the internal dynamo can be expressed using Eqs. (17) and (35) as

$B_{\phi, \text { dyn }}=C_{2} \epsilon \gamma_{\text {dyn }}^{1 / 2} \alpha_{\mathrm{ss}}^{1 / 20} \bar{\mu}^{3 / 16} M^{7 / 16} y^{17 / 40} R^{-21 / 16}$,

where

$C_{2}=\left(\mu_{0} C_{1}\right)^{1 / 2}$.

The magnetic field due to the shear can be written as:

$B_{\phi, \text { shear }}=\frac{\mu \gamma}{R^{3}}\left[1-\left(\frac{R}{R_{\mathrm{c}}}\right)^{3 / 2}\right]$,

where

$R_{\mathrm{c}}=\left(\frac{G M P_{\mathrm{spin}}^{2}}{4 \pi^{2}}\right)^{1 / 3} \simeq 1.5 \times 10^{6} P_{\mathrm{spin}}^{2 / 3} M_{1}^{1 / 3} \mathrm{~m}$

is the corotation radius, at which the Keplerian angular velocity is the same as the stellar angular velocity. Here $P_{\text {spin }}=2 \pi / \Omega_{\mathrm{s}}$ is the spin period of the star, and $M_{1}=M / M_{\odot}$. Equations (37) and (39) show that the magnetic field generated by the internal dynamo varies more slowly with radius $\sim R^{-1.3}$ than the magnetic field due to shear, $\sim R^{-3}$. Thus the dynamo component dominates at large radii.

Equation (21) gives us an ordinary differential equation for $y$

$$
\begin{array}{r}
y^{\prime}=\frac{\dot{M}}{6 \pi R}-\frac{y}{2 R}-C_{3} y^{17 / 40} R^{-45 / 16} \\
-C_{4} R^{-9 / 2}\left[1-\left(\frac{R}{R_{\mathrm{c}}}\right)^{3 / 2}\right]
\end{array}
$$

where

$C_{3}=\frac{4}{3 \mu_{0} \sqrt{G}} C_{2} \epsilon \gamma_{\mathrm{dyn}}^{1 / 2} \alpha_{\mathrm{ss}}^{1 / 20} M^{-1 / 16} \bar{\mu}^{3 / 16} \mu$

and

$C_{4}=\frac{4 \gamma}{3 \mu_{0} \sqrt{G}} M^{-1 / 2} \mu^{2}$

The solution of Eq. (41) approaches the Shakura-Sunyaev solution at large radii, thus giving us the boundary condition $y \longrightarrow \frac{\dot{M}}{3 \pi}$ as $R \longrightarrow \infty$.

We introduce the dimensionless variable $\Lambda$ through

$y=\Lambda \dot{M}$

and a dimensionless radial coordinate through

$R=r R_{\mathrm{A}}$,

where $R_{\mathrm{A}}$ is the Alfvén radius,

$R_{\mathrm{A}}=\left(\frac{2 \pi^{2} \mu^{4}}{G M \dot{M}^{2} \mu_{0}^{2}}\right)^{1 / 7}=5.1 \times 10^{6} \dot{M}_{13}^{-2 / 7} M_{1}^{-1 / 7} \mu_{20}^{4 / 7} \mathrm{~m}$,

which is given by putting the magnetic pressure equal to the ram pressure of the accreting fluid (e.g. Frank et al. 2002). Here $\dot{M}_{13}$ represents the mass transfer rate in units of $10^{13} \mathrm{~kg} \mathrm{~s}^{-1}$, and $\mu_{20}$ is the stellar magnetic dipole moment in units of $10^{20} \mathrm{~T} \mathrm{~m}^{3}$. The boundary condition is then $\Lambda \longrightarrow \frac{1}{3 \pi}$ as $r \longrightarrow \infty$, and Eq. (41) can be written as

$\Lambda^{\prime}=-\frac{\Lambda}{2 r}+\frac{1}{6 \pi r}-C_{5} \Lambda^{17 / 40} r^{-45 / 16}-C_{6} r^{-9 / 2}\left(1-\omega_{\mathrm{s}} r^{3 / 2}\right)$

where

$C_{5}=4.9 \epsilon \alpha_{\mathrm{ss}}^{1 / 20} \gamma_{\mathrm{dyn}}^{1 / 2} \bar{\mu}^{3 / 16} M_{1}^{11 / 56} \dot{M}_{13}^{-2 / 35} \mu_{20}^{-1 / 28}$,

$C_{6}=0.3 \gamma$,

and

$\omega_{\mathrm{s}}=\left(\frac{R_{\mathrm{A}}}{R_{\mathrm{c}}}\right)^{3 / 2}=6.3 \dot{M}_{13}^{-3 / 7} M_{1}^{-5 / 7} \mu_{20}^{6 / 7} P^{-1}$

is the fastness parameter.

\section{Numerical solution}

\subsection{Global solutions}

We integrate Eq. (47) inwards from a large radius, usually $100 R_{\mathrm{A}}$, to which we impose the boundary condition that $\Lambda=\frac{1}{3 \pi}$, which is similar to the approach by Brandenburg \& Campbell (1998), but most studies of accretion discs have rather applied a boundary condition at the inner edge of the disc, and then integrated the equations outwards.

There are two possible boundary conditions that can be applied at the inner edge of the accretion disc, either that $\Lambda=0$, which corresponds to that $\rho$ and $T \rightarrow 0$ at the inner edge (case D), or that

$\frac{\mathrm{d}}{\mathrm{d} r}\left(r^{3} \Lambda \frac{\mathrm{d} \Omega}{\mathrm{d} r}\right)=0$

which means that the viscosity does not at all contribute to driving the accretion at this radius. Case D is the boundary condition 
Table 1. Spin parameters of the models.

\begin{tabular}{lll}
\hline \hline$P(\mathrm{~s})$ & $R_{\mathrm{c}}(\mathrm{m})$ & $\omega_{\mathrm{s}}$ \\
\hline 7 & $6.1 \times 10^{6}$ & 0.71 \\
18.7 & $1.2 \times 10^{7}$ & 0.26 \\
100 & $3.6 \times 10^{7}$ & 0.05 \\
\hline
\end{tabular}

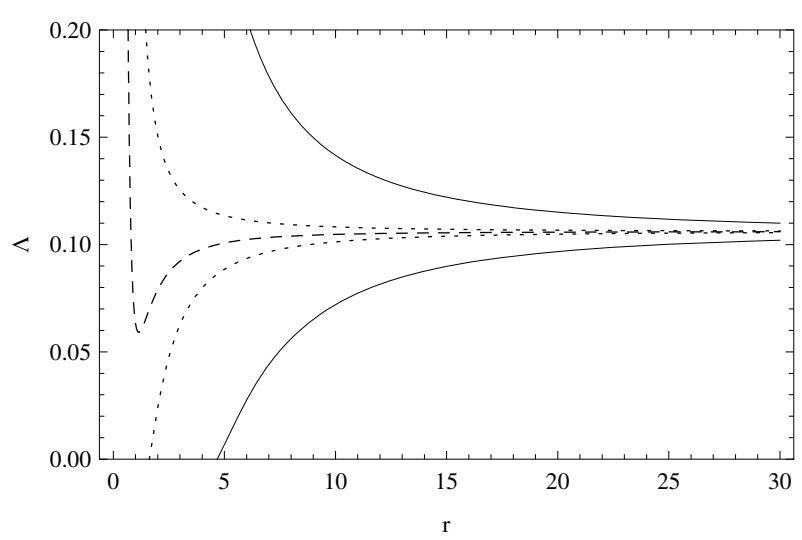

Fig. 1. $\Lambda(r)$ for our fiducial neutron star with a spin period of $7 \mathrm{~s}$ and $\epsilon=1,0.1,0,-0.1,-1$ from the top to the bottom.

that has been most widely used and that was adopted for instance by Shakura \& Sunyaev (1973). In this case the density drops to zero because the inflow velocity becomes infinite, which is of course not realistic, and a more accurate treatment shows that the inflow becomes transonic close to this position Paczyński \& Bisnovatyi-Kogan (1981). In case V the inflow at the inner edge of the accretion disc is driven completely by the transfer of excess angular momentum from the accreting matter to the stellar magnetic field (e.g. Wang 1995).

For our fiducial model, we take a neutron star of $M=1.4 M_{\odot}$ and a magnetic moment of $10^{20} \mathrm{~T} \mathrm{~m}^{3}$, which is accreting at $10^{13} \mathrm{~kg} \mathrm{~s}^{-1}$. The dimensionless parameters $\gamma$ and $\gamma_{\text {dyn }}$ are, respectively, 1 and 10 in our fiducial model, while $\alpha_{\mathrm{ss}}=0.01$. The exact values of $\alpha_{\mathrm{ss}}$ and $\gamma_{\mathrm{dyn}}$ are unimportant, since we vary the parameter $\epsilon$ below, but $\gamma$ influences the solutions in its own way as is shown at the end of this section. We consider three different spin periods with corresponding corotation radii and fastness parameters (see Table 1). The system goes into the propeller regime for $\omega_{\mathrm{s}} \geq 1$ (e.g. Illarionov \& Sunyaev 1975; Ghosh \& Lamb 1979a,b).

Firstly, for a spin period of $7 \mathrm{~s}$ and $\epsilon=1,0.1,0,-0.1$, and -1 , we get the solutions shown from the top to the bottom of Fig. 1. The $\epsilon=-1$ and -0.1 solutions have case $D$ inner boundaries at 4.7 and $1.6 R_{\mathrm{A}}$, respectively, while the other three solutions have case $\mathrm{V}$ inner boundaries (Fig. 2). The inner boundary is close to $R_{\mathrm{A}}$ if $\epsilon=0$, which corresponds to the absence of an internal disc dynamo, but moves outwards as $|\epsilon|$ increases. In case $\mathrm{V}$, the solution continues inside the inner edge of the accretion disc, but the viscosity counteracts the accretion, which is instead driven by the magnetic stresses. This regime has been described by Campbell (1998), who discusses how the disc is disrupted in this region, we assume that this region belongs to a boundary layer that we do not attempt to model in this paper. On the other hand, all solutions approach the Shakura-Sunyaev solution at large radii, as required by our boundary condition.

By increasing the spin peroid to $100 \mathrm{~s}$, we see that the $\epsilon=$ -0.1 solution also develops a case V inner boundary (Fig. 3), but there are only small quantitative changes for the $\epsilon=-1$ and +1 solutions. (The inner boundary of the $\epsilon=-1$ solution

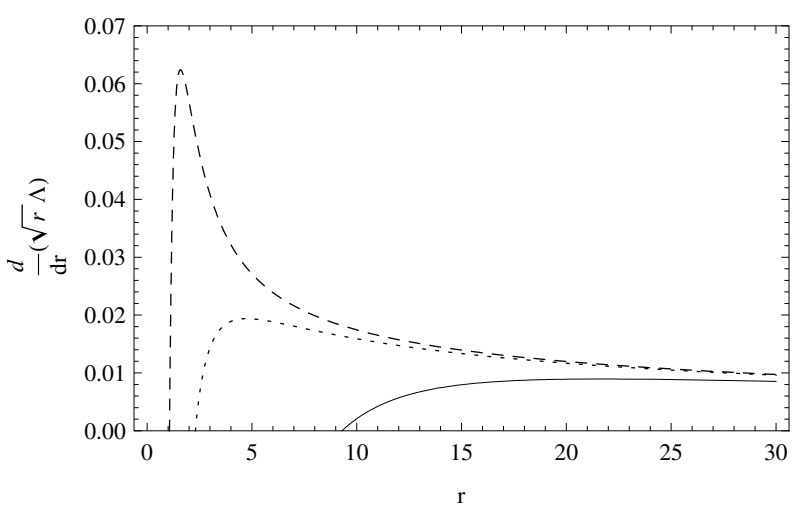

Fig. 2. $\frac{\mathrm{d}}{\mathrm{d} r}(\sqrt{r} \Lambda)$, as a function of $r$ for the fiducial neutron star with a spin peroid of $7 \mathrm{~s}$ and with $\epsilon=1,0.1$, and 0 from the bottom to the top.

is now located at $4.4 R_{\mathrm{A}}$.) The dependence of the solution on the spin period can be better seen in Fig. 4, where we vary the spin period, when $\epsilon$ is fixed to 0 . Then $\Lambda$ has a local minimum for $P_{\mathrm{s}}=7 \mathrm{~s}$, but this minimum weakens and disappears as the spin period is increased. This is similar to how $\Lambda$ depends on $\epsilon$. For $\epsilon=-1, \Lambda$ has an unphysical negative local minima at a small radius, such that the physical solution has a case D boundary at several Alfvén radii. As $\epsilon$ is increased, the local minimum grows and the solution develops a case $\mathrm{V}$ inner boundary when the minimum becomes positive. Increasing $\epsilon$ removes the local minimum further and the solution becomes a strictly decreasing function of $r$, which asymptotically approaches $1 / 3 \pi$ as required by our boundary condition. Increasing $\gamma$ has a similar effect to decreasing $\epsilon$ (Fig. 5).

\subsection{The structure of an accretion disc}

The physical structure of the accretion disc can now be expressed using $\Lambda$ and $r=R / R_{\mathrm{A}}$. We have that

$$
\begin{aligned}
& \Sigma=3.7 \times 10^{3} \alpha_{\mathrm{ss}}^{-4 / 5} M_{1}^{5 / 14} \dot{M}_{13}^{32 / 35} \mu_{20}^{-3 / 7} \Lambda(r)^{7 / 10} r^{-3 / 4} \mathrm{~kg} \mathrm{~m}^{-2} \\
& T_{\mathrm{c}}=4.8 \times 10^{5} \alpha_{\mathrm{ss}}^{-1 / 5} \bar{\mu}^{1 / 4} M_{1}^{5 / 14} \dot{M}_{13}^{18 / 35} \mu_{20}^{-3 / 7} \Lambda(r)^{3 / 10} r^{-3 / 4} \mathrm{~K} \\
& \frac{H}{R}=0.012 \alpha_{\mathrm{ss}}^{-1 / 10} \bar{\mu}^{-3 / 8} M_{1}^{-11 / 28} \dot{M}_{13}^{4 / 35} \mu_{20}^{1 / 14} \Lambda(r)^{3 / 20} r^{1 / 8}
\end{aligned}
$$

$$
\begin{aligned}
& \rho=3.0 \times 10^{-2} \alpha_{\mathrm{ss}}^{-7 / 10} \bar{\mu}^{9 / 8} M_{1}^{25 / 28} \dot{M}_{13}^{38 / 35} \mu_{20}^{-15 / 14} \Lambda(r)^{11 / 20} \\
& \times r^{-15 / 8} \mathrm{~kg} \mathrm{~m}^{-3} \\
& \tau=370 \alpha_{\mathrm{ss}}^{-4 / 5} \bar{\mu} \dot{M}_{13}^{1 / 5} \Lambda(r)^{1 / 5} \\
& v=2.7 \times 10^{9} \alpha_{\mathrm{ss}}^{4 / 5} \bar{\mu}^{-3 / 4} M_{1}^{-5 / 14} \dot{M}_{13}^{3 / 35} \mu_{20}^{3 / 7} \Lambda(r)^{3 / 10} r^{3 / 4} \mathrm{~m}^{2} \mathrm{~s}^{-1} \\
& v_{R}=84 \alpha_{\mathrm{ss}}^{4 / 5} \bar{\mu}^{-3 / 4} M_{1}^{-3 / 14} \dot{M}_{13}^{13 / 35} \mu_{20}^{-1 / 7} \Lambda(r)^{-7 / 10} r^{-1 / 4} \mathrm{~m} \mathrm{~s}^{-1} \\
& B_{\phi, \text { dyn }}=12 \epsilon \gamma_{\text {dyn }}^{1 / 2} \alpha_{\mathrm{ss}}^{1 / 20} \bar{\mu}^{3 / 16} M_{1}^{5 / 8} \dot{M}_{13}^{4 / 5} \mu_{20}^{-3 / 4} \Lambda(r)^{17 / 40} r^{-21 / 16} \mathrm{~T} \\
& B_{\phi, \text { shear }}=0.75 \gamma M_{1}^{3 / 7} \dot{M}_{13}^{6 / 7} \mu_{20}^{-5 / 7} r^{-3}\left(1-\omega_{\mathrm{s}} r^{3 / 2}\right) \mathrm{T} .
\end{aligned}
$$

In Fig. 6 we show the surface density as a function of radius for the fiducial neutron star with a spin peroid of $7 \mathrm{~s}$ and $\epsilon=-1,0$, and 1 , respectively. For $\epsilon=-1$ the surface density attains a local maximum, while the other models have surface densities that are strictly decreasing functions of $r$. Figure 7 shows the radial 


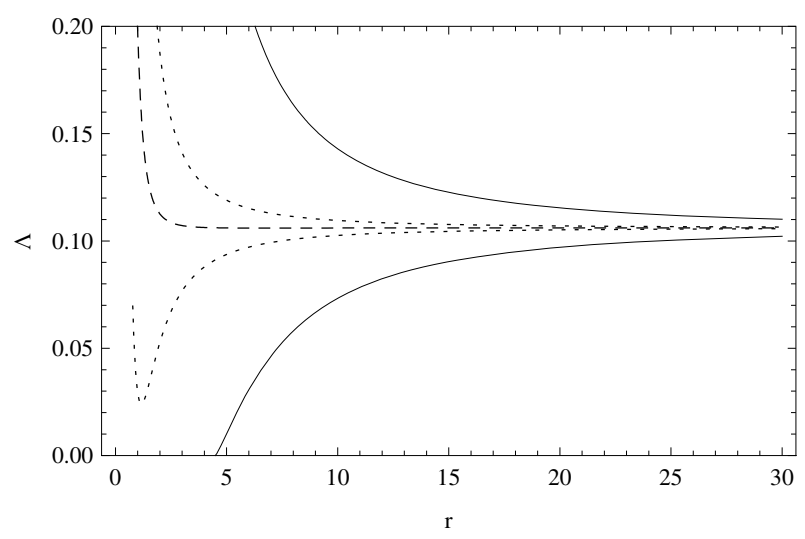

Fig. 3. $\Lambda(r)$ for our fiducial neutron star with a spin period of $100 \mathrm{~s}$ and $\epsilon=1,0.1,0,-0.1,-1$ from the top to the bottom.

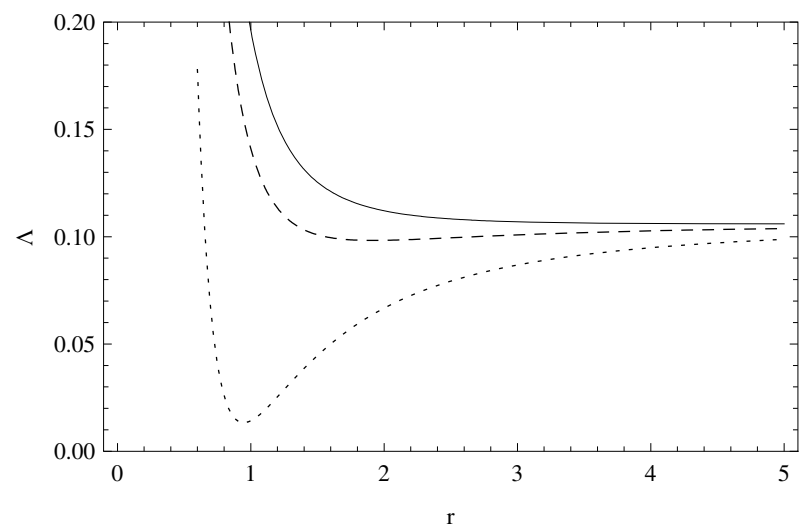

Fig. 4. $\Lambda(r)$ for our fiducial neutron star with $\epsilon=0$ and spin periods of $100,18.7$, and $7 \mathrm{~s}$ from the top to the bottom.

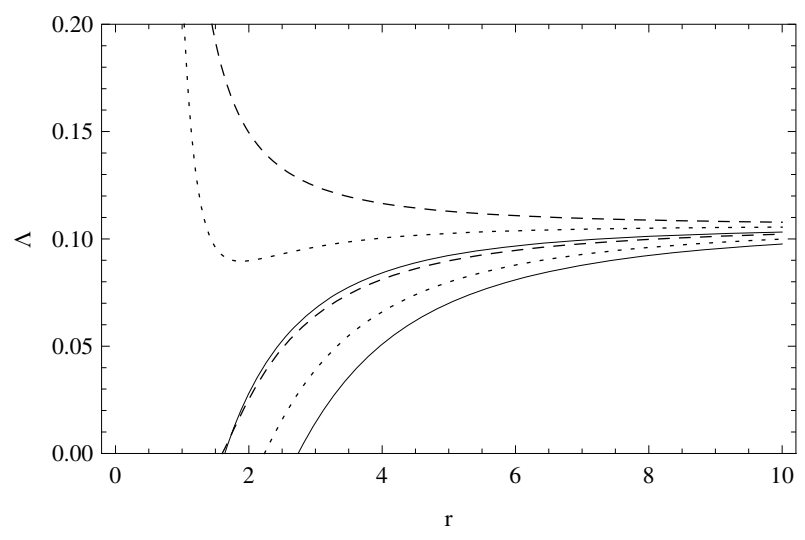

Fig. 5. $\Lambda(r)$ for our fiducial neutron star with spin period of $7 \mathrm{~s}$ and $\epsilon=0.1$ and $\gamma=1,3$, and 5 (upper dashed, dotted, and solid lines, respectively), $\epsilon=-0.1$ and $\gamma=1,3$, and 5 (lower dashed, dotted, and solid lines, respectively).

velocity as a function of $r$. Since $v_{R} \propto \Sigma^{-1}$, it becomes infinite as the inner edge of the disc for $\epsilon=-1$, but it stays finite for $\epsilon=0$ and 1 and eventually goes to 0 in the boundary layer. Increasing the spin period of the neutron star has a very marginal effect on the accretion disc, though $T_{\mathrm{c}}$, which is proportional to $\Sigma^{2}$, increases somewhat (Fig. 8).

We plot the magnetic fields for our fiducial model with varying $\epsilon$ and spin periods of 7 and 100 s, respectively, in Figs. 9 and 10 , respectively. The corotation radius, at which $B_{\phi \text { shear }}$ changes sign, occurs inside of the inner edge of the accretion

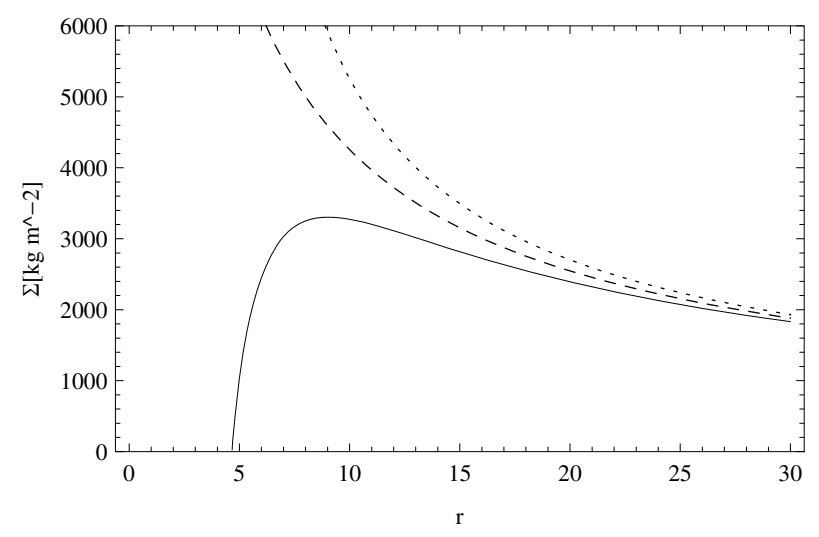

Fig. 6. $\Sigma$ as a function of $r$ for the fiducial neutron star with a spin period of $7 \mathrm{~s}$ and $\epsilon=-1$ (solid line), $\epsilon=0$ (dashed line), and $\epsilon=1$ (dotted line).

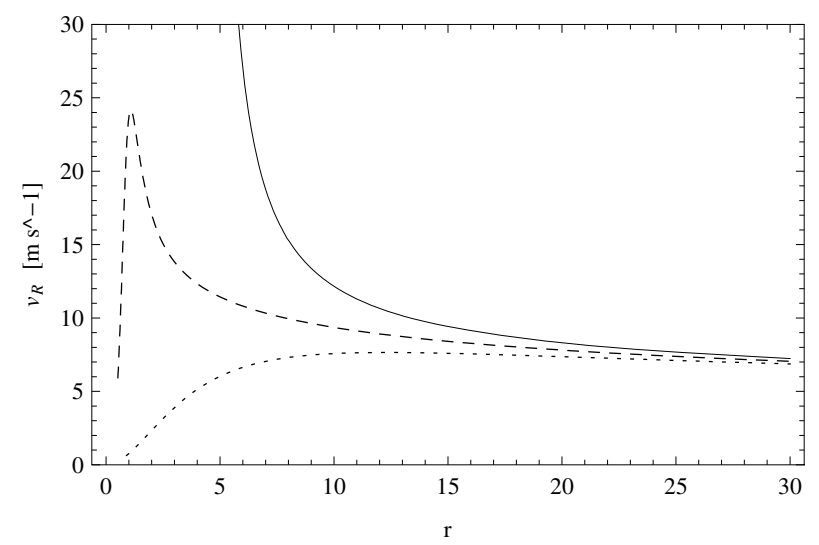

Fig. 7. $V_{R}$ as a function of $r$ for the fiducial neutron star with a spin period of $7 \mathrm{~s}$ and $\epsilon=-1$ (solid line), $\epsilon=0$ (dashed line), and $\epsilon=1$ (dotted line).

disc for $\epsilon=1$ and -1 , and for these values of $\epsilon, B_{\phi \operatorname{dyn}}$ is the dominant magnetic field everywhere inside the disc.

In general the ratio of the magnetic field due to shear to that generated by the internal dynamo is

$$
\begin{aligned}
\frac{B_{\phi \text { shear }}}{B_{\phi \text { dyn }}}= & 6.25 \times 10^{-2} \epsilon^{-1} \gamma \gamma_{\mathrm{dyn}}^{-1 / 2} \alpha_{\mathrm{ss}}^{-1 / 20} \bar{\mu}^{-3 / 16} M_{1}^{-11 / 56} \dot{M}_{13}^{2 / 35} \mu_{20}^{1 / 28} \\
& \times\left(1-\omega_{\mathrm{s}} r^{3 / 2}\right) \Lambda(r)^{-17 / 40} r^{-27 / 16} .
\end{aligned}
$$

It is only during rather extreme conditions that the shear-induced field can dominate at small radii, and the dynamo is always dominant at large radii.

\section{Discussion}

\subsection{The inner edge of the accretion disc}

We have shown that there are two different forms of inner disc boundaries that can be found among our solutions. We denote these as case D and V, respectively. Case D occurs only for sufficiently negative $\epsilon$, while case $V$ covers a larger part of the parameter domain that we have studied. We summarise our values for the inner radius of the accretion disc in Table 2. We see that the inner radius can be significantly larger than the Alfvén radius when $|\epsilon|$ is close to unity. This is the result of the dynamo enhancing the coupling of the stellar magnetic field to the accretion flow, such that it dominates the viscous torque at larger radii than would otherwise be the case. 
S. B. Tessema and U. Torkelsson: The structure of thin accretion discs around magnetised stars

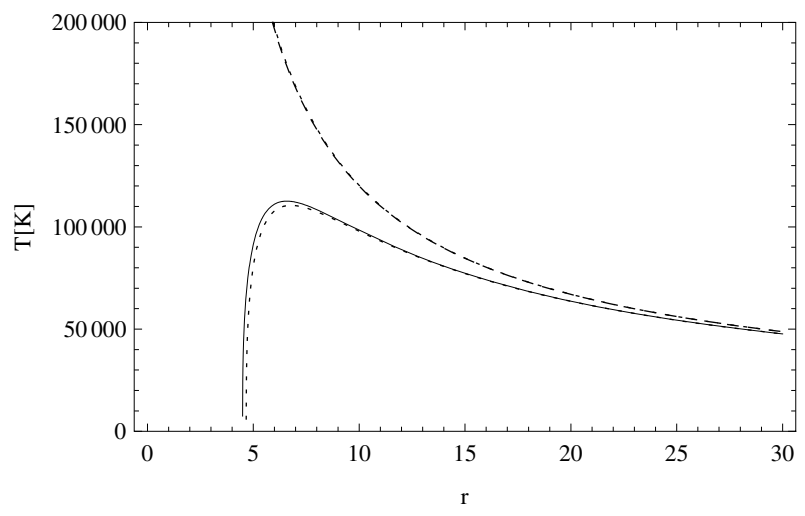

Fig. 8. $T_{\mathrm{c}}$ as a function of $r$. The two lower curves show discs with $\epsilon=-1$ around neutron stars with spin periods of $7 \mathrm{~s}$ (dashed line) and $100 \mathrm{~s}$ (solid line), respectively. The two upper curves show discs with $\epsilon=1$ around neutron stars with spin periods of $7 \mathrm{~s}$ (dashed line) and $100 \mathrm{~s}$ (dotted line), respectively.

\subsection{The angular momentum balance}

To understand the exchange of angular momentum between the accretion disc and its environment we multiply Eq. (21) by $2 \pi R$ and integrate it from $R_{0}$, the inner radius of the disc, to $R_{1}$ the outer edge of the disc

$$
\begin{aligned}
& -\dot{M}\left(\sqrt{G M R_{1}}-\sqrt{G M R_{0}}\right)=\int_{R_{0}}^{R_{1}} 4 \pi \frac{B_{z} B_{\phi, \mathrm{dyn}}}{\mu_{0}} R^{2} \mathrm{~d} R \\
& +\int_{R_{0}}^{R_{1}} 4 \pi \frac{B_{z} B_{\phi, \text { shear }}}{\mu_{0}} R^{2} \mathrm{~d} R-3 \pi(v \Sigma)_{R_{1}} \sqrt{G M R_{1}} \\
& +3 \pi(v \Sigma)_{R_{0}} \sqrt{G M R_{0}} .
\end{aligned}
$$

The lefthand side is the difference between the angular momentum that is advected out of the inner edge of the accretion disc and that which is fed into the disc at its outer edge and the righthand side describes the contribution of magnetic and viscous torques to the angular momentum balance. The term $3 \pi(v \Sigma)_{R_{1}} \sqrt{G M R_{1}}$ describes the viscous tension at the outer edge of the disc, which is not considered further in this paper. Rather, we consider the exchange of angular momentum between the accretion disc and the neutron star. Firstly, we have the angular momentum which is advected from the disc to the star

$N_{\mathrm{adv}}=\dot{M} \sqrt{G M R_{0}}=2.6 \times 10^{26} \mu_{20}^{2 / 7} M_{1}^{3 / 7} \dot{M}_{13}^{6 / 7} r_{0}^{1 / 2}$.

Secondly, we have the magnetic torques on the neutron star, which we divide into one part due to the shear

$$
\begin{aligned}
N_{\mathrm{shr}}= & -\int_{R_{0}}^{R_{1}} 4 \pi \frac{B_{z} B_{\phi, \text { shear }}}{\mu_{0}} R^{2} \mathrm{~d} R \\
= & -7.5 \times 10^{26} \gamma \mu_{20}^{2 / 7} M_{1}^{3 / 7} \dot{M}_{13}^{6 / 7} \\
& \times \int_{\mathrm{r}_{0}}^{\infty}\left[r^{-4}\left(1-\omega_{\mathrm{s}} r^{3 / 2}\right)\right] \mathrm{d} r
\end{aligned}
$$

and a second part due to the dynamo

$$
\begin{aligned}
N_{\mathrm{dyn}}= & -\int_{R_{0}}^{R_{1}} 4 \pi \frac{B_{z} B_{\phi, \mathrm{dyn}}}{\mu_{0}} R^{2} \mathrm{~d} R \\
= & 1.2 \times 10^{28} \epsilon \gamma_{\mathrm{dyn}}^{1 / 2} \alpha_{\mathrm{ss}}^{1 / 20} \bar{\mu}^{3 / 16} \mu_{20}^{1 / 4} M_{1}^{5 / 8} \dot{M}_{13}^{4 / 5} \\
& \times \int_{\mathrm{r}_{0}}^{\infty} \Lambda^{17 / 40} r^{-37 / 16} \mathrm{~d} r .
\end{aligned}
$$

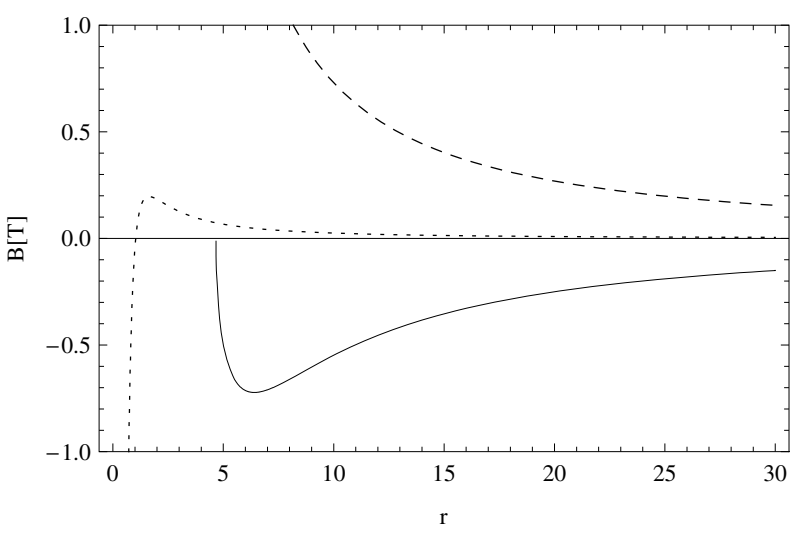

Fig. 9. The toroidal magnetic field for the fiducial neutron star with a spin period of $7 \mathrm{~s}$. The solid and dashed lines show the field generated by the dynamo for $\epsilon=-1$ and 1, respectively, while the dotted line shows the magnetic field generated by the shear.

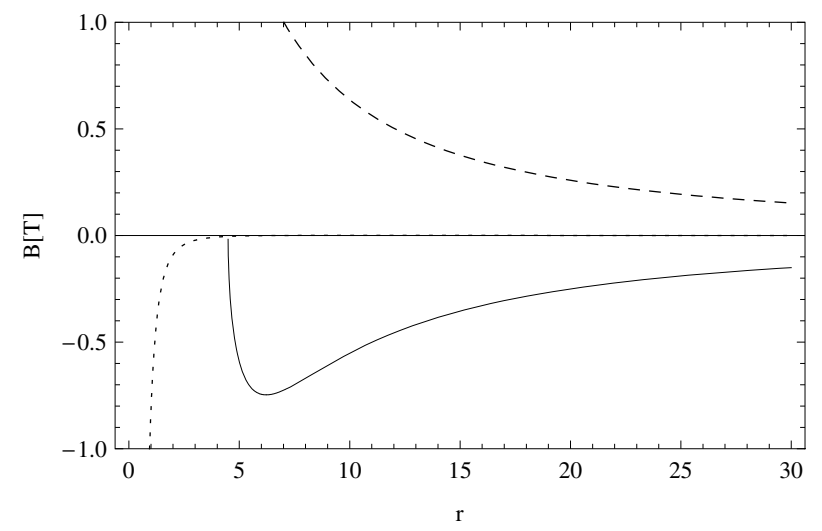

Fig. 10. The toroidal magnetic field for the fiducial neutron star with a spin period of $100 \mathrm{~s}$. The solid and dashed lines show the field generated by the dynamo for $\epsilon=-1$ and 1 , respectively, while the dotted line shows the magnetic field generated by the shear.

Finally the viscous stress at the inner edge of the accretion disc transports angular momentum outwards away from the neutron star resulting in a torque

$N_{\text {vis }}=-3 \pi(v \Sigma)_{R_{0}} \sqrt{G M R_{0}}=-2.5 \times 10^{27} \mu_{20}^{2 / 7} M_{1}^{3 / 7} \dot{M}_{13}^{6 / 7} \Lambda r_{0}^{1 / 2}$.

This torque vanishes for a case D inner boundary. We can now calculate the torques on our fiducial neutron star for our choices of spin periods and $\epsilon$. These results are summarised in Table 2. Unless $\epsilon=0$ we see that the magnetic torque due to the dynamo is always significantly stronger than the magnetic torque due to the shear, and both are stronger for $\epsilon=0.1$ than for $\epsilon=1$. The reason for this effect is that the central hole in the disc grows too large when $\epsilon=1$. The dominant torque at $\epsilon=1$ is therefore the viscous torque at the inner boundary, which has usually been ignored.

Let us now compare our results with the BATSE data (Bildsten et al. 1997). The 7.6s X-ray pulsar 4U1626-67 was observed to spin down at a rate $\dot{v} \approx-7 \times 10^{-13} \mathrm{~Hz} \mathrm{~s}^{-1}$ and spin up at $\dot{v} \approx+8.5 \times 10^{-13} \mathrm{~Hz} \mathrm{~s}^{-1}$. These spin variations correspond to torques

$N=2 \pi \dot{v} I=6.3 \times 10^{25} \dot{v}_{-13} I_{38} \mathrm{Nm}$,

where $I$ is the moment of inertia of the neutron star, which we measure in $10^{38} \mathrm{~kg} \mathrm{~m}^{2}$, and we measure the spin acceleration in 
Table 2. The inner edge of the accretion disc and its torque on the fiducial neutron star.

\begin{tabular}{lllllllll}
\hline \hline$P_{\text {spin }}[\mathrm{s}]$ & $\epsilon$ & Case & $R_{0}$ & $N_{\text {shear }}$ & $N_{\text {dyn }}$ & $N_{\text {adv }}$ & $N_{\text {vis }}$ & $N_{\text {tot }}$ \\
\hline 7 & 1 & $\mathrm{~V}$ & $9.0 R_{\mathrm{A}}$ & $7.0 \times 10^{24}$ & $5.7 \times 10^{26}$ & $7.8 \times 10^{26}$ & $-1.2 \times 10^{27}$ & $2.6 \times 10^{26}$ \\
& 0.1 & $\mathrm{~V}$ & $2.0 R_{\mathrm{A}}$ & $5.3 \times 10^{25}$ & $4.3 \times 10^{27}$ & $3.7 \times 10^{26}$ & $-4.9 \times 10^{26}$ & $4.2 \times 10^{27}$ \\
& 0 & $\mathrm{~V}$ & $1.0 R_{\mathrm{A}}$ & $5.9 \times 10^{25}$ & 0 & $2.6 \times 10^{26}$ & $-1.2 \times 10^{26}$ & $2.0 \times 10^{26}$ \\
& -0.1 & $\mathrm{D}$ & $1.6 R_{\mathrm{A}}$ & $6.3 \times 10^{25}$ & $-3.7 \times 10^{27}$ & $3.3 \times 10^{26}$ & 0 & $-3.3 \times 10^{27}$ \\
& -1 & $\mathrm{D}$ & $4.7 R_{\mathrm{A}}$ & $1.8 \times 10^{25}$ & $-9.1 \times 10^{26}$ & $5.6 \times 10^{26}$ & 0 & $-3.2 \times 10^{26}$ \\
18.7 & 1 & $\mathrm{~V}$ & $9 R_{\mathrm{A}}$ & $2.4 \times 10^{24}$ & $5.7 \times 10^{26}$ & $7.8 \times 10^{26}$ & $-1.1 \times 10^{27}$ & $2.5 \times 10^{26}$ \\
& 0.1 & $\mathrm{~V}$ & $2 R_{\mathrm{A}}$ & $8.2 \times 10^{24}$ & $4.4 \times 10^{27}$ & $3.7 \times 10^{26}$ & $-5.4 \times 10^{26}$ & $4.2 \times 10^{27}$ \\
& 0 & $\mathrm{~V}$ & $1.0 R_{\mathrm{A}}$ & $-6.7 \times 10^{25}$ & 0 & $2.6 \times 10^{26}$ & $-3.0 \times 10^{26}$ & $1.7 \times 10^{26}$ \\
& -0.1 & $\mathrm{~V}$ & $1.0 R_{\mathrm{A}}$ & $-6.7 \times 10^{25}$ & $6.0 \times 10^{27}$ & $2.6 \times 10^{26}$ & $-2.5 \times 10^{25}$ & $-5.9 \times 10^{27}$ \\
& -1 & $\mathrm{D}$ & $4.6 R_{\mathrm{A}}$ & $6.0 \times 10^{24}$ & $-9.3 \times 10^{26}$ & $5.6 \times 10^{26}$ & 0 & $-3.7 \times 10^{26}$ \\
& 1 & $\mathrm{~V}$ & $10 R_{\mathrm{A}}$ & $2.9 \times 10^{23}$ & $4.9 \times 10^{26}$ & $8.2 \times 10^{26}$ & $-1.0 \times 10^{27}$ & $2.8 \times 10^{26}$ \\
& 0.1 & $\mathrm{~V}$ & $2.5 R_{\mathrm{A}}$ & $-5.4 \times 10^{24}$ & $3.2 \times 10^{27}$ & $4.1 \times 10^{26}$ & $-6.2 \times 10^{26}$ & $3.0 \times 10^{27}$ \\
& 0 & $\mathrm{~V}$ & $1.2 R_{\mathrm{A}}$ & $-7.1 \times 10^{25}$ & 0 & $2.9 \times 10^{26}$ & $-4.4 \times 10^{26}$ & $-2.3 \times 10^{26}$ \\
& -0.1 & $\mathrm{~V}$ & $1.0 R_{\mathrm{A}}$ & $-1.3 \times 10^{26}$ & $-7.1 \times 10^{27}$ & $2.6 \times 10^{26}$ & $-4.5 \times 10^{25}$ & $-6.9 \times 10^{27}$ \\
& -1 & $\mathrm{D}$ & $4.4 R_{\mathrm{A}}$ & $-1.4 \times 10^{23}$ & $-9.4 \times 10^{26}$ & $5.5 \times 10^{26}$ & 0 & $-4.0 \times 10^{26}$ \\
\hline
\end{tabular}

units of $10^{-13} \mathrm{~Hz} \mathrm{~s}^{-1}$. The required torque is significantly larger than what is produced by $N_{\text {shear }}$ in any of our models, but $N_{\text {dyn }}$ is even an order of magnitude greater than this value if $|\epsilon|=0.1$. This overestimate can be explained by that we have assumed a unidirectional $B_{\phi, \text { dyn }}$ across the disc surface, while it might be more realistic to expect that the toroidal field is organised in magnetic annuli with opposite polarities, or that $|\epsilon|$ is significantly smaller fraction of the total turbulent magnetic field.

We explain the torque reversals in the same way as in Torkelsson (1998), where the dynamo undergoes a field reversal, which in our model corresponds to $\epsilon$ changing sign. One might speculate that, during this field reversal, the disc passes through a state corresponding to $\epsilon=0$, in which the disc will have a smaller inner radius than during the states with an active dynamo. The closer to the surface of the neutron star that the accretion disc extends, the more of the X-ray emission from the accretion columns it can absorb and re-process. Torkelsson (1998) showed that the observed time scales on which the torques remain constant in the sources Cen X-3, OAO 1657-415, and GX $1+4$ are comparable to the global viscous time scales of their accretion discs, which constrains the mechanism that is responsible for the reversals of the magnetic field.

\section{Conclusions}

We have investigated the interaction between a magnetic neutron star and its surrounding accretion disc in the case where the accretion disc is supporting an internal dynamo. The magnetic field that is produced by the dynamo can lead to a significant enhancement of the magnetic torque between the neutron star and the accretion disc, compared to what is seen in the model by Ghosh \& Lamb (1979a,b). This extra magnetic torque can explain the large variations in spin frequencies of Cen X-3 and OAO 1657-415 (Bildsten et al. 1997). Furthermore, a reversal of the magnetic field that is generated by the dynamo, similar to the reversals that we see of the magnetic fields on the Sun, could explain the torque reversals in these objects.

From the way that we calculate the structure of the accretion disc, we find two kinds of solutions with different behaviours at the inner edge. A few of our solutions have case D boundaries at which the density and temperature go to 0 at finite radius, while most of our solutions have case $\mathrm{V}$ boundaries at which the accretion is driven entirely by the magnetic tension between the accreting matter and the neutron star. In this case there is a viscous stress between the accretion disc and the boundary layer, which can transfer angular momentum outwards at a rate that is comparable to the one at which it is advected inwards by the accreting matter itself.

We have also found that the dynamo leads to that the inner edge of the accretion disc occurs at a radius that is larger than the traditional Alfvén radius. This effect is weak, though, for a realistic value of the dynamo-generated magnetic field.

Acknowledgements. S.B.T. thanks the Department of Physics at the University of Gothenburg for hospitality and support during this project. SBT is supported in part by the Swedish Institute (SI) under their Guest Scholarship Programme. UT thanks the Department of Physics at Addis Ababa University for their hospitality. This research has made use of NASA's Astrophysics Data System. We thank an anonymous referee for comments that have improved the quality of the paper.

\section{References}

Balbus, S. A., \& Hawley, J. F. 1991, ApJ, 376, 214

Balbus, S. A., \& Hawley, J. F. 1998, Rev. Mod. Phys., 70, 1

Bardou, A., \& Heyvaerts, J. 1996, A\&A, 307, 1009

Bildsten, L., Chakrabarty, D., Chiu, J., et al. 1997, ApJS, 113, 367

Brandenburg, A., \& Campbell, C. G. 1998, MNRAS, 298, 223

Brandenburg, A., Nordlund, A., Stein, R. F., \& Torkelsson, U. 1995, ApJ, 446, 741

Campbell, C. G. 1997, Magnetohydrodynamics in binary stars (Dordrecht: Kluwer)

Campbell, C. G. 1998, MNRAS, 301, 754

Campbell, C. G., \& Heptinstall, P. M. 1998a, MNRAS, 299, 31,

Campbell, C. G., \& Heptinstall, P. M. 1998b, MNRAS, 301, 558

Frank, J., King, A. R., \& Raine, D. 2002, Accretion power in astrophysics (Cambridge University Press)

Ghosh, P., \& Lamb, F. K. 1979a, ApJ, 232, 259

Ghosh, P., \& Lamb, F. K. 1979b, ApJ, 234, 296

Hawley, J. F., Gammie, C. F., \& Balbus, S. A. 1995, ApJ, 440, 742

Illarionov, A. F., \& Sunyaev, R. A. 1975, A\&A, 39, 185

Lightman, A. P., \& Eardley, D. M. 1974, ApJ, 187, L1

Nelson, R. W., Bildsten, L., Chakrabarty, D., et al. 1997, ApJ, 488, L117

Paczyński, B., \& Bisnovatyi-Kogan, G. 1981, Acta Astron., 31, 283

Parmar, A. N., White, N. E., Stella, L., Izzo, C., \& Ferri, P. 1989, ApJ, 338, 359

Shakura, N. I., \& Sunyaev, R. A. 1973, A\&A, 24, 337

Torkelsson, U. 1998, MNRAS, 298, L55

Wang, Y. M. 1995, ApJ, 449, L153 